\title{
Against Pointillisme: a Call to Arms
}

\author{
Jeremy Butterfield
}

Monday 12 July 2010: Forthcoming in

Explanation, Prediction and Confirmation: New Trends and Old Ones Reconsidered Edited by: D. Dieks, W. Gonzalez, S. Hartmann, T.Uebel and M. Weber; to be published by Springer

\begin{abstract}
This paper forms part of a wider campaign: to deny pointillisme. That is the doctrine that a physical theory's fundamental quantities are defined at points of space or of spacetime, and represent intrinsic properties of such points or point-sized objects located there; so that properties of spatial or spatiotemporal regions and their material contents are determined by the point-by-point facts.

Elsewhere, I argued against pointillisme about chrono-geometry, and about velocity in classical mechanics. In both cases, attention focussed on temporal extrinsicality: i.e. on what an ascription of a property implies about other times. Therefore, I also discussed the metaphysical debate whether persistence should be understood as endurance or perdurance.

In this paper, I focus instead on spatial extrinsicality: i.e. on what an ascription of a property implies about other places. The main idea will be that the classical mechanics of continuous media (solids or fluids) involves a good deal of spatial extrinsicality - which seems not to have been noticed by philosophers, even those who have no inclination to pointillisme.

I begin by describing my wider campaign. Then I present some elementary aspects of stress, strain and elasticity - emphasising the kinds of spatial extrinsicality they each involve.

I conduct the discussion entirely in the context of "Newtonian" ideas about space and time. But my arguments carry over to relativistic physics.
\end{abstract}




\section{Contents}

1 Introduction 1

1.1 Five kinds of spatial extrinsicality . . . . . . . . . . . . . . . 2

2 The wider campaign against pointillisme 5

2.1 Avoiding controversy about the intrinsic-extrinsic distinction . . . . . . 5

2.1.1 Distinction from three mathematical distinctions . . . . . . . . . 5

2.2 Classical mechanics is not pointilliste . . . . . . . . . . . . 6

2.2.1 Two versions of pointillisme . . . . . . . . . . . 6

2.2.2 Mechanics violates pointillisme as regards space . . . . . . . . 7

3 Stress: extrinsicality from a tensor's order $\quad 8$

3.1 The need for surface forces . . . . . . . . . . . . . . . . . . . 9

3.2 The stress tensor . . . . . . . . . . . . . . . . . . . . . . 10

4 Strain: haecceitist kinematics $\quad 12$

4.1 The description of deformations . . . . . . . . . . . . . 13

4.2 The Cauchy-Green strain tensor . . . . . . . . . . . . . . . . . . . . . 13

5 Elasticity: further along the spectrum of extrinsicality 14

6 Conclusion: yet more extrinsicality 15

$\begin{array}{lll}7 & \text { References } & 17\end{array}$

\section{Introduction}

This paper forms part of a wider campaign: to deny pointillisme. That is the doctrine that a physical theory's fundamental quantities are defined at points of space or of spacetime, and represent intrinsic properties of such points or point-sized objects located there; so that properties of spatial or spatiotemporal regions and their material contents are determined by the point-by-point facts.

I will first describe this wider campaign (Section 2). The broad picture is that we should distinguish between temporal extrinsicality, i.e. what an ascription of a property implies about other times, and spatial extrinsicality, i.e. what an ascription of a property implies about other places. Elsewhere, I have proclaimed the former (2004, 
2005, 2006a, 2006b). That is, I argued that there is more temporal extrinsicality than the pointilliste admits. This led me to the metaphysical debate whether the persistence of objects over time should be understood as endurance or as perdurance; (and in particular to assessing the so-called 'rotating discs argument' (2006a)).

But here, I will proclaim spatial extrinsicality: the way that an ascription "reaches across space" like extended arms - hence my punning title. My main point will be that classical continuum mechanics gives us many rich examples of spatial extrinsicality: which seem never to have been noticed in analytic metaphysics, even though the relevant physics goes back to Euler. (I will confine myself to the "Newtonian" conception of space and time, as applied to continuum mechanics. This restriction keeps things simple: and at no cost, since both the debate and my arguments carry over to relativistic physics, once one there adopts a space vs. time distinction.)

This paper also forms part of a yet wider and more positive topic, which is of interest to philosophers who feel no attraction for pointillisme. Namely: the philosophical richness of classical mechanics, especially continuum mechanics. This went without saying for the natural philosophers - later divided into physicists and philosophers of nature! - from the time of Newton to about 1910. Thereafter, the conceptual aspects, indeed problems, of the revolutionary quantum and relativity theories grabbed centrestage in the philosophy of nature. But the richness of classical mechanics remains, as emphasized in recent decades by such authors as Truesdell, in mechanics and its history, and Mark Wilson in philosophy (1998, 2006, Chapters 4 to 6). I have no space here, even for a list of topics; but my (2004, Section 2; 2004a, Section 2; 2004b, Section 3f.) give some discussion and references. Here, I just note that even as regards pointillisme, this paper is more positive than its predecessors, in two ways. The second is more important, and deserves a separate Subsection.

First, one main focus of the earlier papers was whether properties of a point (or point-sized object) that are represented by vectors, tensors, connections etc. can be intrinsic to it. Typically, pointillistes try to reconcile pointillisme with the fact that such properties certainly seem to be extrinsic. So in those papers, I argued that their proposed reconciliations fail. (One main aspect was that their reconciliations involved heterodox construals of the properties. So besides criticizing the reconciliations on their own terms, I urged that once pointillisme was rejected, there was less motivation for heterodoxy.) One really does need extrinsic properties of points, both for chronogeometry (my 2006) and for the concept of velocity in mechanics (2006b). But in this paper I have no pointilliste authors as targets, for the simple reason that the spatial extrinsicality at work in continuum mechanics has not been noticed by metaphysicsand so no one has proposed a pointilliste reconciliation that I need to rebut.

\subsection{Five kinds of spatial extrinsicality}

We will see that continuum mechanics exhibits kinds of extrinsicality which are more varied and richer than the temporal extrinsicality which was centre-stage in the earlier 
papers. There, temporal extrinsicality related to two main topics:

(i) implications about the existence of the same particle at different times, and so the endurance-perdurance debate (especially 2004, 2005, 2006a); and

(ii) the idea of a spectrum of implications, arising from the sequence of successively higher time-derivatives of position (2006a, Section 4.5; 2006b, Sections 3.2, 4.3).

Here in this paper, spatial extrinsicality will not relate to (i). There will of course be an analogue of (ii): a spectrum of extrinsicality due to the sequence of successively higher spatial derivatives. Indeed, there is "more extrinsicality", since one has to consider derivatives not just of position, but also of many other quantities. But more important: we need to recognize that continuum mechanics also involves several other kinds of spatial extrinsicality. This paper will be mostly concerned with a kind which, like (ii), involves a spectrum. This spectrum arises, not from higher derivatives, but from geometric structures such as lines and planes through the spatial point in question (and the angles between them). Taking successively more lines and-or planes through the point yields successively higher-order tensors: another sense of "more extrinsicality". Sections 3, 4 and 5 will give examples. (For simplicity, I will consider only rectilinear coordinates: I just note that curvilinear coordinates would give another source of extrinsicality.)

Space prevents my going into details about continuum mechanics' other kinds of spatial extrinsicality. So let me just list, and then set aside, the following three.

(1) "Measure": In treating quantities like mass and charge, continuum mechanics takes a leaf from modern measure theory (cf e.g. Truesdell 1991, pp. 16-19, 92-94). It attributes a quantity like mass primarily to finite volumes, and takes mass density as a derived quantity, defined by a limiting process. This means the mass-density at a spatial point is spatially extrinsic; (even though it is a scalar quantity-which some discussions wrongly take to imply intrinsicality: cf. Section 2.1.1). Besides, such densities are only defined almost everywhere, in the measure-theoretic sense: making the spatial extrinsicality subtle (cf. my 2006, Sections 3.3.2.B and 4.6).

(2) "Metric": I have also set aside relativity's description of continuous matter (or fields). Here, the idea of mass is replaced by a energy-momentum tensor whose definition depends on the spacetime metric in various subtle ways. This suggests that mass-energy, momentum, stress etc. are best seen as relations of the matter (or field) to spacetime structure. Lehmkuhl (2010) is an excellent discussion of this sort of extrinsicality.

(3) "Topology": Facts about the global topology of space, or spacetime, or of configuration space, can induce another, perhaps more striking, kind of extrinsicality. For it is natural to respond to the kinds already listed by saying that, though they no doubt refute pointillisme beyond repair, surely they allow some doctrine of "localism", that takes the bearers of a physical theory's fundamental quantities to be, not pointillisme's points (or point-sized bits of matter), but rather: arbitrarily small spatial or spacetime regions (or correspondingly small bits of matter). Thus localism might be defined as claiming that the state assigned by a physical theory to (the systems within) a spa- 
tial or spacetime region $R$ is determined by (supervenient upon) the states assigned to the elements of a covering of $R$ consisting of arbitrarily small open sets. And this looks to be compatible with the kinds of extrinsicality so far listed. (In particular, my own earlier proclamation of temporal extrinsicality proposed such a localism as appropriate for an anti-pointilliste defence of perdurance as an account of persistence: 2006a, Section 4.) But in electromagnetism, global topological facts can lead to phenomena (most famously, the Aharonov-Bohm effect) which seem best interpreted in terms of holonomies (values of integrals around loops), thus violating localism. (Myrvold (2010) is an excellent discussion: what I have dubbed 'localism', he calls 'patchy separability'. $)^{1}$

To sum up: continuum classical mechanics involves at least five kinds of spatial extrinsicality. Namely: the three kinds (1) to (3) just listed, and the two I first mentioned:

(4) "Derivatives": The spectrum of extrinsicality due to successively higher spatial derivatives;

(5) "Orders": The spectrum of extrinsicality due to successively higher order tensors. $^{2}$

This paper will focus on (5). Indeed, most of my points will flow from the fact that continuum mechanics embraces spatial extrinsicality due to surface forces, acting at a point, with respect to a surface through that point. In Section 3, this idea will be encapsulated in the stress tensor. (In short, stress is a codification of the forces acting at a point.) Besides, by considering successively higher order tensors, we get a spectrum of extrinsicality. This is illustrated in Section 5 by elasticity. Elasticity relates stress to strain: which, in short, is a codification of how a continuous body is distorted at the point. So as a preliminary to discussing elasticity, I need to discuss strain, in particular the strain tensor. I do this in Section 4: where we see yet another philosophical aspect of classical mechanics - that it treats the identity of particles as primitive in the sense of distinguishing a configuration and its permuted image, even if the permutation shifts only indistinguishable particles.

\footnotetext{
${ }^{1}$ You might object that electromagnetism is a different theory than continuum mechanics, so that presumably the latter is free of this kind of extrinsicality. I think this objection is valid only in letter, not in spirit. Without going into details, I would say, as regards 'the letter': though mechanics does invoke holonomies in explanations, I concede that, at least so far as I know, holonomies are not interpretatively essential to mechanics, in the way they seem to be for electromagnetism. But as to 'the spirit': continuum mechanics is not a conceptually closed theory, since the treatment of many topics, such as a body's restorative forces or its expansion under heat, inevitably leads to other theories of classical physics, such as thermodynamics and electromagnetism - as the Chapter titles of any of the more comprehensive treatises attest. So when interpreting continuum mechanics, it is artificial - indeed, merely a unconscious reflection of a common pedagogic restriction - to set aside these adjacent theories. Indeed the holonomy interpretation of the classical electromagnetic field is similarly based on considering adjacent theories; in that case, the quantum mechanics of a particle in such a field. For more discussion, cf. e.g. my (2004, Section 2.2), and Belot (1998, Section 5).

${ }^{2}$ Although I here have no space to relate these kinds to each other, it is clear that this would be a good project.
} 


\section{The wider campaign against pointillisme}

My earlier papers described this wider campaign, in particular how it relates to the debate whether persistence should be understood as endurance or perdurance. So here I shall just say, and in part repeat, what little is needed for later Sections.

\subsection{Avoiding controversy about the intrinsic-extrinsic distinc- tion}

My campaign does not need to take sides in the ongoing controversy about how to analyse, indeed understand, the intrinsic-extrinsic distinction. In this paper, I can make do with a much clearer distinction, between a species of extrinsic property that Lewis (1983, p. 114) dubbed 'positive extrinsic', and the rest.

The positive extrinsic properties are those that imply accompaniment, where something is accompanied iff it coexists with some wholly distinct contingent object. So the negation of this is: properties that are compatible with being unaccompanied, i.e. being the only contingent object in the universe (for short: being lonely). Lewis pointed out (against a proposal of Kim's) that, since loneliness is itself obviously extrinsic, this distinction is not the same as the intrinsic-extrinsic distinction; though 'almost any extrinsic property that a sensible person would ever mention is positive extrinsic' (1983, p. 115). But this distinction is enough for me. That is, I can take pointillisme to advocate properties that are intrinsic in the weak sense of being not positively extrinsic (i.e. being compatible with loneliness). So this makes my campaign's claims, i.e. my denial of pointillisme, logically stronger; and so I hope more interesting. Anyway, my campaign makes some novel proposals about positive extrinsicality. As we saw in Section 1, I distinguish temporal and spatial (positive) extrinsicality; and in this paper, I will propose degrees of (positive) spatial extrinsicality.

\subsubsection{Distinction from three mathematical distinctions}

Both (a) the murky intrinsic-extrinsic distinction, and (b) the clearer distinction between positive extrinsics and the rest, are different distinctions from three that are made within mathematics and physics, especially in those parts relevant to us: viz. pure and applied differential geometry. The first of these distinctions goes by the name 'intrinsic'/'extrinsic'; the second is called 'scalar'/'non-scalar', and the third is called 'local'/'non-local'. Though my previous papers used only the third distinction, this paper will use the second and third. These distinctions are as follows.

(i): The use of 'intrinsic' in differential geometry is a use which is common across all of mathematics: a feature is intrinsic to a mathematical object if it is determined (defined) by just the object as given, without appeal to anything extraneous - in particular a coordinate system, or an embedding of the object into another. For example, 
we say that the intrinsic geometry of a cylinder is flat; it is only as embedded in $\mathbb{R}^{3}$ that it is curved.

(ii): Differential geometry classifies quantities according to how they transform between coordinate systems: the simplest case being scalars which have the same value in all coordinate systems. We will not need the algebraic details of how the other cases - vectors, tensors, connections etc.- transform. But we will need some basic ideas about the geometric ideas that underly the transformation rules. For these are all about choosing appropriate lines and planes through the point in question - e.g. three orthogonal axes to define a local coordinate system - by which to give numerical and so algebraic expression to the quantity (vector or tensor etc.). So the geometric ideas behind the transformation rules are the source of the spectrum of spatial extrinsicality announced in Section 1.1. Besides, according to (i)'s mathematical usage, the choice of lines and planes through a point is extrinsic to it. So here (i)'s usage of 'extrinsic', and my philosophical usage, mesh well; (though in general the philosophical distinctions, both (a) and (b), are different from the three mathematical ones).

(iii): Differential geometry uses 'local' (as vs. 'global') in various ways. But the central use is that a mathematical object or structure is local if it is associated with a point by being determined (defined) by the structures defined on any neighbourhood, no matter how small, of the point. For example, the instantaneous velocity of a pointparticle at a spacetime point, and all the higher derivatives of its velocity, are local since their existence and values are determined (in the usual way as a limit of quotients of differences) by the particle's trajectory in an arbitrarily small neighbourhood of the point. Similarly, for countless other examples of a derivative, or higher derivative, of a quantity at a point. This suggests that these derivatives represent an extrinsic property of the point, even if the original quantity was intrinsic to it. I agree. And my previous papers discussed:

(i): how the sequence of derivatives suggests a spectrum of extrinsicality; and

(ii): how the derivative's being mathematically local makes the extrinsicality very mild: to take again the example of velocity, one might say that instantaneous velocity, acceleration and all higher derivatives, are 'hardly extrinsic'.

\subsection{Classical mechanics is not pointilliste}

\subsubsection{Two versions of pointillisme}

I should distinguish a weaker and a stronger version of pointillisme. They differ by:

(i) the weaker version taking 'point' in pointillisme to mean spatial point; and so denying spatial extrinsicality;

(ii) the stronger version taking 'point' in pointillisme to mean spacetime point; and so denying temporal as well as spatial extrinsicality.

Previous papers rebutted the stronger version; (this involved rebutting the rotating discs argument, so that one can understand persistence as perdurance). Here I rebut 
the weaker one.

Thus I take 'pointillisme as regards space' to be, roughly, the doctrine that the instantaneous state of the world is fully described by all the intrinsic properties, at that time, of all spatial points and-or point-sized bits of matter. Here, 'intrinsic' means 'spatially intrinsic'. That is, attributing such a property to an object carries no implications about spatially distant objects; but it can carry implications about objects (perhaps temporal parts of the given object) at other times.

On the other hand: I take 'pointillisme as regards spacetime' to be, roughly, the doctrine that the history of the world is fully described by all the intrinsic properties of all the spacetime points and-or all the intrinsic properties at all the various times of point-sized bits of matter (either point-particles, or in a continuum). And here 'intrinsic' means just the negation of Lewis' 'positive extrinsic'. So it means 'both spatially and temporally intrinsic': attributing such a property carries no implications about objects at other places, or at other times.

It is of course a delicate matter to relate either version of pointillisme to the content of specific physical theories. One naturally asks for example, how philosophers' idea of an intrinsic property relates to the idea of a physical quantity. Here my tactic will be the obvious one: to formulate pointillisme as a doctrine relativized to (i.e. as a property of) a given physical theory. Thus I will take this paper's target, pointillisme as regards space, to be a trio of claims about a physical theory, as follows.

(a): the fundamental quantities of the theory are defined at points of space;

(b): these quantities represent intrinsic properties of such points;

(c): models of the theory - i.e. in physicists' jargon, solutions of its equations, and in metaphysicians' jargon, possible worlds according to the theory - are fully defined by a specification of the quantities' values at all spatial points for all the various times.

So, putting (a)-(c) together: the idea is that the theory's models (or solutions or worlds) are something like conjunctions or mereological fusions of "ultralocal facts", i.e. facts at points. Similarly, pointillisme as regards spacetime would be a trio of claims, but substituting 'spacetime points' for 'spatial points'.

\subsubsection{Mechanics violates pointillisme as regards space}

Though I have not made a systematic survey, there is no doubt that pointillisme, as regards either space or spacetime, is prominent in recent analytic metaphysics of nature, especially of neo-Humean stripe. The prime example is the metaphysical system of David Lewis, which is so impressive in its scope and detail: one of his main metaphysical theses, 'Humean supervenience' (1986, pp. ix-xvi; 1994), is a version of pointillisme as regards spacetime. More specifically, I think many metaphysicians believe that classical physics - or at least classical mechanics - satisfies pointillisme, at least as regards space; (though perhaps not as regards spacetime, because of the rotating discs argument).

I agree that there are some considerations in favour of this belief, even the stronger 
belief about all of classical physics not just classical mechanics. Most of the quantities of most classical physical theories are defined at points of space or spacetime, with models being defined by those quantities' values at the various points. Witness the endemic use of functions on space or spacetime, governed by differential equations. This supports (a) and (c) in pointillisme's defining trio of claims. Besides, classical physics is free of various kinds of "holism", and so anti-pointillisme, that are suggested by entanglement in quantum theory; (as is often remarked: e.g. Teller (1986), Healey (1991, p. 408f.), Esfeld (2001, Chapter 8)). I say 'kinds' because the basic fact of entanglement - that the pure state of a composite system (a "whole") is not determined by the states of its components ("parts") — manifests differently in different settings: e.g. (i) in the frame-dependent description of systems in relativity (Myrvold 2002, 2003, 2009), (ii) in heterodox versions of quantum theory such as pilot-wave theory (e.g. Holland 1993, Chapter 7).

Nevertheless, this belief in pointillisme is false, even just for classical mechanics. The flaw lies in claim (b). I already mentioned in Section 1.1 five reasons why (b) is falsefive kinds of spatial extrinsicality; (though one reason, viz. my (3), applied in the first instance to branches of classical physics other than mechanics). As I announced there, I will not develop all these reasons: I will just concentrate on one which, though very familiar in the mechanics textbooks, has (as far as I know) been wholly ignored in the philosophical literature. Namely, continuum mechanics embraces spatial extrinsicality due to surface forces, acting at a point, with respect to a surface through that point. We will first see this in Section 3's discussion of the stress tensor. After that, by considering successively higher order tensors, we will get a spectrum of extrinsicality. I emphasize that all the formalism to follow was established by figures such as Euler and Cauchy between 1770 and 1825; though I shall report it in modern guise, in particular using vector calculus. ${ }^{3}$

So to sum up: my complaint against the widespread metaphysical view that classical mechanics is pointilliste as regards space is that, although pointillisme's claims (a) and (c) mostly hold good for classical mechanics, claim (b) is endemically false. Furthermore, one main reason why it is false was known already by 1825 - but seems to have been wholly ignored in the philosophical literature.

\section{Stress: extrinsicality from a tensor's order}

In all that follows, I must be brief about technicalities. So I will assume the ideas of tensor algebra and tensor calculus on $\mathbb{R}^{3}$, taken as our model of physical space. But I need only consider orthonormal bases and reference frames, and so can suppress the distinction between contravariance and covariance (upper and lower indices): I will use lower indices. More generally, I will follow the exposition, and level of rigour, of

\footnotetext{
${ }^{3}$ For the history of vectors and vector calculus, cf. Crowe (1967). I stress again that this physics was not overturned by the advent of relativity: although I will ignore relativity, all my arguments apply equally to relativistic continuum mechanics.
} 
Gonzalo and Stuart's ${ }^{4}$ excellent text (2008): where the details I must suppress, and much more, can be found. (The same material is treated with more rigour (e.g. about measure theory; cf. (1) in Section 1.1) in texts such as Gurtin (1981), Marsden and Hughes (1983) and Truesdell (1991).)

I will also "let pointillisme off the hook" about the foundations of geometry. That is, I will allow that the pointilliste can invoke any ideas and results of geometry, in particular of tensor algebra and tensor calculus, as legitimately as the rest of us. But this allowance is a matter of not flogging a dead horse, rather than conviction: my (2006) argues that the pointilliste has grave trouble with geometry, in particular tensor calculus.

From now on, the basic idea will be that a continuous body is placed in physical space $\mathbb{R}^{3}$ by occupying a suitable set $B \subset \mathbb{R}^{3}{ }^{5} B$ is called the placement or configuration of the body. I will not be precise about how to define 'suitable set'. Suffice it to make two comments: the first technical, the second philosophical. (i): The main technical desideratum is that the meaning of 'suitable' should enable one to secure the invaluable integral theorems of vector calculus (such as the divergence and Stokes' theorems); and so one usually requires $B$ to consist of a finite number (usually one!) of open bounded disjoint components, and for the boundary $\partial B$ to be piecewise smooth and orientable; (cf. e.g. Gonzalo and Stuart (2008, p. 54) or Kellogg (1967)). (ii): Unfortunately, most philosophical discussion of what 'suitable' should mean has been divorced from (i), and dominated by traditional verbal conundrums about whether two continuous bodies can touch each other: Smith (2007) is a fine attempt to connect the philosophical discussion to the physics.

Building on this basic idea, this Section considers the forces exerted on the (material points constituting the) body at points $x \in B$. The next Section considers the distortion of the body.

\subsection{The need for surface forces}

We begin with a type of force that pointillisme can accommodate: more precisely, can accommodate once we set aside the other kinds of spatial extrinsicality listed in Section 1.1! Namely, body forces. These are forces exerted on the body's material points at a distance, and so without regard to any contact between parts of the body, or between the body and its environment. The archetypal example is gravity. We model such a force with a continuous (or at least: suitably integrable) function on $B$. Thus the body force field, per unit volume, exerted by an external influence on $B$ is a vector-valued function $b: B \rightarrow V$. (We will write $V$, rather than $\mathbb{R}^{3}$, for the (unique!) 3-dimensional real vector space, when it is not being interpreted as physical space.) So the resultant force on a sub-volume $\Omega \subset B$ is: $r_{b}(\Omega)=\int_{\Omega} b(x) d V_{x}$.

\footnotetext{
${ }^{4}$ Whom I thank for permission to reproduce Figures 1 to 3 below.

${ }^{5}$ The Newtonian model of physical space is sometimes taken to be the affine space $\mathrm{E}^{3}$ (' $\mathrm{E}$ ' for Euclid), rather than $\mathbb{R}^{3}$. But for simplicity, I "keep the origin" and use $\mathbb{R}^{3}$.
} 
Similarly for the body force's influence on rotational motion, as encapsulated in the idea of torque. I shall not go into details about the need for this idea. Suffice it to say that continuum mechanics needs the idea of the torque on $\Omega$ about a point $z$, given by: $\tau_{b}(\Omega)=\int_{\Omega}(x-z) \times b(x) d V_{x}$. And under our present (charitable!) assumptions, pointillisme can no doubt accommodate the torque $\tau_{b}(\Omega)$ just as well as $r_{b}(\Omega)$.

But continuum mechanics also needs the idea of a surface force. Physically, this is a force that arises by contact; and its mathematical representation requires relativizing the force to the surface along which there is contact. This latter idea is fairly intuitive, when the surface in question is the body's boundary and the force is exerted on the body at the surface by the environment (or vice versa): this is called an 'external surface force'.

But in order to describe how a body resists being broken up by forces that vary across its spatial extent, continuum mechanics also needs internal surface forces. Indeed, it needs to consider at any point $x$ within $B$, with respect to an arbitrary surface $\Gamma$ through $x$, the surface force exerted by the material on one side of $\Gamma$, on the material on the other side. Nowadays, textbooks take this idea in their stride. That may seem fair enough, since after all, under small enough forces, a distorted body does not break up along any internal surface; so presumably the description of the body will need some sort of balance of forces across every such surface. But prima facie, the idea is puzzling: how can a force exerted on an internal material point "pull on the point with respect to" a surface? And certainly, to consider all such surfaces through all internal points amounts to a major expansion of the framework for describing forces, compared to a body force's resultant force and torque, $\tau_{b}(\Omega)$ and $r_{b}(\Omega)$. So it is unsurprising that historically, it was a major struggle to articulate the need for this idea. (It is no doubt also unsurprising that the first person to realize this need, and the expanded framework's sufficiency for describing continua, was that monumental genius, Euler - in the 1770s: for details, cf. e.g. Casey (1992), Wilson 1998 (Section 6).)

History aside, the mathematical representation of internal surface forces is given by the Euler-Cauchy Cut Principle, as follows. Let us write $\hat{n}: \Gamma \rightarrow V$ for the unit normal field on an arbitrary oriented surface $\Gamma$. Then the Principle postulates that the internal surface force per unit area on $\Gamma$ is represented by the surface force field (also called traction field) $t_{\hat{n}}: \Gamma \rightarrow V$. As with body forces, we can define a resultant force on $\Gamma$ by integration: it is $r_{s}(\Gamma):=\int_{\Gamma} t_{\hat{n}}(x) d A_{x}$. Here, the main point for us is that the subscript $\hat{n}$ indicates that the function's value for a point $x \in \Gamma$ depends on the surface $\Gamma$ : the nature of this dependence will be our next topic, in Section 3.2.

\subsection{The stress tensor}

In this Section, a natural simplifying assumption about how the traction field $t_{\hat{n}}$ depends on the surface $\Gamma$ will imply a simple and powerful representation of the field: viz. the stress tensor. This assumption is called 'Cauchy's postulate'. When taken together with two mild continuity assumptions, it implies an analogue, for the traction field, of 
Newton's Third Law (that action and reaction are equal and opposite). This is often called 'Cauchy's Lemma'. Then Cauchy's Lemma yields (with no further assumptions) Cauchy's Theorem: that the traction field at any point is encoded by a second-order tensor, the (Cauchy) stress tensor. This is, essentially, a $3 \times 3$ matrix with the usual elementary rules for transforming under a change of basis vectors.

Cauchy's postulate says that the traction field $t_{\hat{n}}$ depends on $\Gamma$ only via its unit normal $\hat{n}$ at the argument-point $x \in \Gamma$. That is: writing $N$ for the unit sphere in $V \equiv \mathbb{R}^{3}$, there is a function $t: N \times B \rightarrow V$, called the traction function for $B$, such that:

$$
t_{\hat{n}}(x)=t(\hat{n}(x), x) .
$$

Thus surfaces $\Gamma_{1}, \Gamma_{2}, \ldots$ that are tangent to each other, with the same normal, at $x$ must have the same traction vector at $x$. Cf. Fig. 1 .

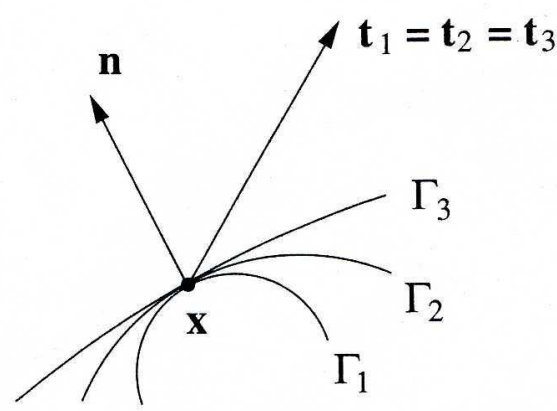

Figure 1: Cauchy's postulate: surfaces through a point $\mathbf{x}$ with normal $\mathbf{n}$ at $\mathbf{x}$ share the same traction vector $\mathbf{t}$ at $\mathbf{x}$.

This is the main assumption required for Cauchy's Lemma.

Cauchy's Lemma: Assume (i) that $t(\hat{n}, x)$ is continuous, and (ii) that the resultant surface force on a body, divided by the surface area, goes to zero as the body's volume tends to zero, i.e. that

$$
\frac{1}{\operatorname{area}(\partial \Omega)} \int_{\partial \Omega} t(\hat{n}(x), x) d A_{x} \rightarrow 0, \text { as } \operatorname{vol}(\Omega) \rightarrow 0
$$

It follows that: $t(-\hat{n}, x)=-t(\hat{n}, x)$. That is: the traction field obeys a law of action and reaction: at each point $x$, the traction exerted by material on one side of a surface $\Gamma$ through $x$ upon the material on the other side is equal and opposite to the traction exerted by the latter material on the former.

This Lemma now yields Cauchy's Theorem, which says that $t$ is given by a secondorder tensor, i.e. a linear map on $V$. (I will denote the set of such linear maps $V \rightarrow V$ by $\operatorname{End}(V)$.)

Cauchy's Theorem: Under these conditions, $t(\hat{n}, x)$ is linear in $\hat{n}$. That is: there is at $x$ a second-order tensor $S(x)$, i.e. a linear map $S(x): V \rightarrow V$, such that $t(\hat{n}, x)=S(x) \hat{n}$. 
Let me put this less abstractly, in terms of components. Recall that the components of a second-order tensor $S: V \rightarrow V$, in any orthonormal frame (i.e. trio of orthogonal unit vectors) $\left(e_{1}, e_{2}, e_{3}\right)$, are defined to be the nine numbers $S_{i j}:=e_{i} \cdot S\left(e_{j}\right)$. One readily checks that Cauchy's Theorem, i.e. $t(\hat{n}, x)=S(x) \hat{n}$, implies that the nine components of $S(x)$ are the components (in the elementary sense for vectors) of the three traction vectors $t\left(e_{j}, x\right)$ for the three coordinate planes through $x$. The ideas underlying this check are that:

(i) a second-order tensor encodes the idea of extracting a real number as a bilinear function of two vectors; and

(ii) a vector in $V \equiv \mathbb{R}^{3}$ is normal to a plane; in particular, each basis vector defines a coordinate plane to which it is normal: and putting (i) and (ii) together, we infer that

(iii) the components of three vectors, each defined with respect to one of three mutually perpendicular planes, can be represented by a second-order tensor. Cf. Fig. 2 .

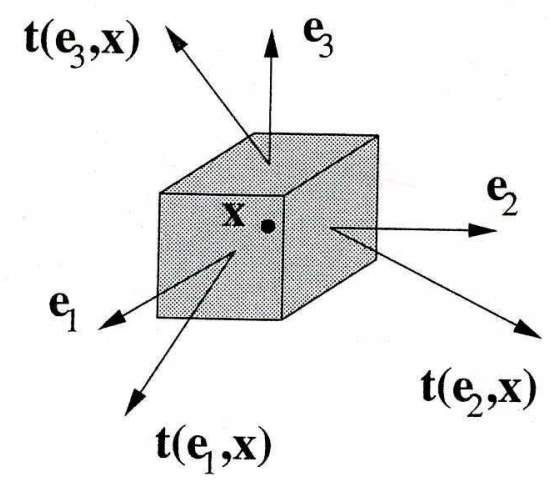

Figure 2: The traction vectors for the coordinate planes with normals $\mathbf{e}_{1}, \mathbf{e}_{2}$ and $\mathbf{e}_{3}$ at $\mathrm{x}$.

The Cauchy stress tensor $S$ is fundamental to all of continuum mechanics; and we will return to it in Section 5. There we will build on this Section's idea that a tensor encodes how a physical quantity can be relative to the lines or planes through a point; and that this implies spatial extrinsicality. More specifically: we will see how going to successively higher-order tensors gives a spectrum of spatial extrinsicality. This will be illustrated by elasticity. But to understand that, we first need the idea of strain.

\section{Strain: haecceitist kinematics}

I now turn from dynamics to kinematics: from forces, that cause a body to accelerate and-or deform, to the geometric description of motion and deformation. This Section has two goals. In Section 4.1, I introduce deformation and related ideas, especially strain. I emphasize how the mathematics takes for granted the underlying identity of 
the body's material points: hence the title's mention of 'haecceitism'. In Section 4.2, I discuss strain. It will be clear that there are various measures of this. For simplicity, I discuss just one: the Cauchy-Green strain tensor.

\subsection{The description of deformations}

At any instant, a body occupies a (suitable) open subset $B$ of $\mathbb{R}^{3}$. The set $B$ does not by itself define the configuration of the body, since it does not specify which material point (infinitesimal point-mass) is at which point within $B$ : think of how rotating a solid ball around its centre gives different configurations with the same occupied sphere.

But having noted this, we will not need to represent in the formalism: we can take the placement of material points at spatial points as understood, and call $B$ a configuration. This is to be compared with some other configuration $B^{\prime}$. We write $X \in$ $\mathbb{R}^{3}$, called material coordinates, for the location of a material point in $B$; and $x \in \mathbb{R}^{3}$, called spatial coordinates, for its location in $B^{\prime}$. Thus we compare the configurations, and keep track of each material point, by $x$ and $X$ being functions of each other: $x=x(X)$ and $X=X(x)$. We call $B$ the reference configuration, and $B^{\prime}$ the deformed configuration; although, as in the ball example, $B^{\prime}$ may have the same shape as (even be the same subset of $\mathbb{R}^{3}$ as) $B$. We focus on $x$ being a function of $X$, rather than vice versa. So we define the deformation map $\phi: B \ni X \mapsto \phi(X):=x \in B^{\prime}$. (This yields the obvious ancillary notion, viz. the displacement field $u: B \rightarrow V \equiv \mathbb{R}^{3}$ defined by $u(X):=\phi(X)-X \in V$. But we will not need this.)

We assume the deformation map $\phi$ is $1-1$, onto, of positive determinant, and smooth. Then we can expect to describe the local nature of a deformation using the deformation gradient (a second-order tensor) $F:=\nabla \phi: X \in B \mapsto \nabla \phi(X) \in \operatorname{End}(V)$. In particular, we focus on the case where $B$ is a small ball, often written as $\Omega$, of radius $\alpha$, and its image $\Omega^{\prime}=\phi(\Omega)$; and we consider the limit as $\alpha \rightarrow 0$. We use 'strain' as a general term to describe the limiting relative differences in the shapes of $\Omega$ and $\Omega^{\prime}$; and so we envisage various measures of strain defined in terms of $F$.

\subsection{The Cauchy-Green strain tensor}

One such measure is the Cauchy-Green strain tensor $C$. It is often useful because (as we shall see) it does not encode information about any overall rotation that there might be in the deformation from $B$ to $B^{\prime}$.

$C$ is defined by multiplying $F$ (on the left) by its transpose $F^{T}$. That is: $C:=$ $F^{T} F: B \rightarrow \operatorname{End}(V)$. To interpret this definition, consider the ball $\Omega$ of radius $\alpha$ around $X \in B$, and any two unit vectors $\hat{e}$ and $\hat{d}$, thus defining points $Y=X+\alpha \hat{e}$ and $Z=X+\alpha \hat{d}$ on the surface of $\Omega$. Let $x, y, z$ denote the corresponding deformed points in $\Omega^{\prime}$, and let $\theta \equiv \theta(\hat{e}, \hat{d})$ be the angle between the vectors $v:=y-x$ and $w:=z-x$. Cf. Figure 3. 


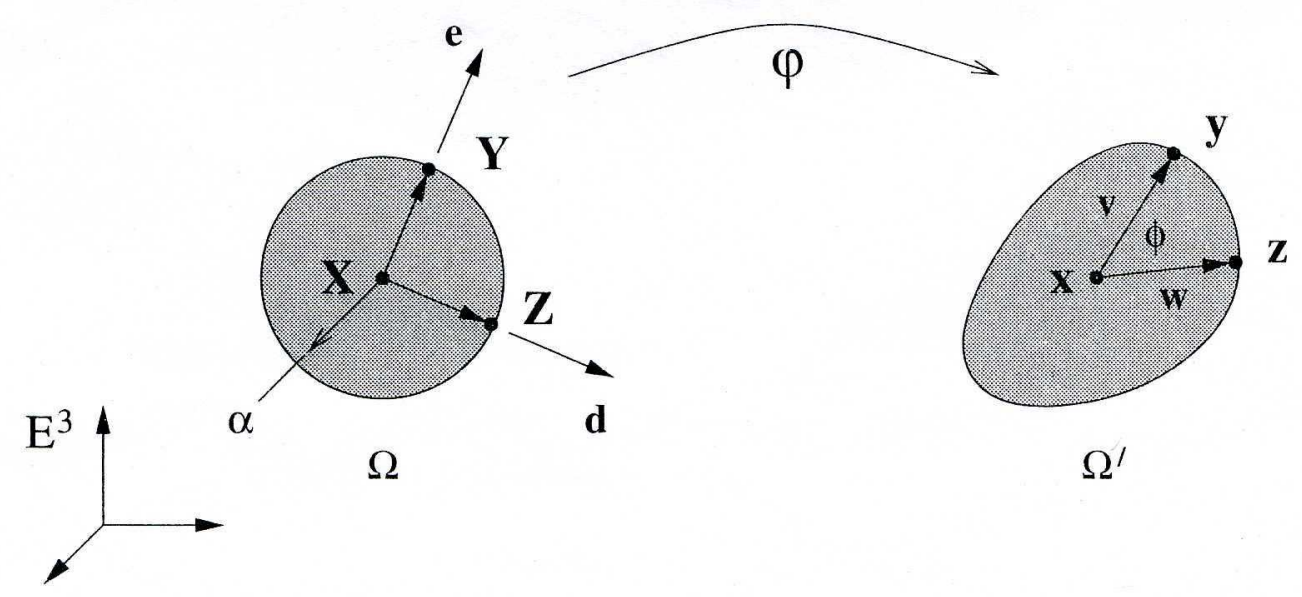

Figure 3: Interpreting the strain tensor $C$. Three points $\mathbf{X}, \mathbf{Y}, \mathbf{Z}$ in $\Omega$ are mapped to corresponding points $\mathbf{x}, \mathbf{y}, \mathbf{z}$ in $\Omega^{\prime}$. $C$ quantifies the limiting changes in the relative position and orientation of the points.

Then it is readily shown that as $\alpha \rightarrow 0$ :

$$
\begin{array}{r}
\frac{|y-x|}{|Y-X|} \rightarrow \sqrt{ }[\hat{e} \cdot C \hat{e}]=: \lambda(\hat{e}), \frac{|z-x|}{|Z-X|} \rightarrow \sqrt{ }[\hat{d} \cdot C \hat{d}]=: \lambda(\hat{d}) ; \text { and } \\
\theta \equiv \cos ^{-1} \frac{v \cdot w}{|v||w|} \rightarrow \cos ^{-1} \frac{\hat{e} \cdot C \hat{d}}{\lambda(\hat{e}) \lambda(\hat{d})}=: \cos ^{-1}(\gamma(\hat{e}, \hat{d})) .
\end{array}
$$

Thus the limiting value $\lambda(\hat{e})$ is called the stretch in the direction $\hat{e}$ at $X$. It is the limit of the ratio of deformed length to initial length for line segments that initially lie along $\hat{e}$ at $X$. And the angle $\gamma(\hat{e}, \hat{d})$ is the limiting value of $\theta$ as $Y$ and $Z$ tend to $X$ along $\hat{e}$ and $\hat{d}$. Denoting the angle between $\hat{e}$ and $\hat{d}$ by $\Theta(\hat{e}, \hat{d})$, the limiting change in angle between these line segments at $X$, i.e. the quantity $\gamma(\hat{e}, \hat{d})-\Theta(\hat{e}, \hat{d})$, is called the shear between $\hat{e}$ and $\hat{d}$ at $X$.

\section{Elasticity: further along the spectrum of extrin- sicality}

We reviewed in Section 3 how a second-order tensor at a point, such as the stress tensor, encodes the idea of extracting a real number as a bilinear function of two vectors there; or equivalently, of giving one vector as a function of another. The emphasis there was on how, since a vector defines (and is defined by) the plane to which it is normal, this corresponds to a function from planes through the point to vectors at it: for the stress tensor, the vector is the traction field (with respect to any surface tangent to the given plane). 
I now turn to the way in which tensor algebra provides a spectrum of spatial extrinsicality, viz. by going to successively higher-order tensors, i.e. multilinear functions of successively more than two vectors. In particular: by combining the ideas of stress and strain, we can find physically important quantities that lie further along this spectrum. Thus the main quantitative relationships between stress and strain are given by a fourth-order tensor - with the appropriate name 'elasticity'. Such a tensor corresponds to extracting a real number from a quadrilinear function of four vectors. The details are as follows.

The main physical idea is simply that stress arises from strain! That is: when we deform a body, putting it under strain, restorative forces arise, preventing it from falling apart (at least for small strains!). The details of exactly what stress arises depend of course on the constitution of the body. In particular, we define an elastic solid as one in which the stress at any point $X \in B$ depends only on the present strain, as encoded by the deformation gradient $F$ at $X$ : not on its rate of change, nor on its past history. This represents a generalization of the familiar Hooke's law, that the force (stress, tension) in a stretched spring is proportional to its extension (strain). Formally: for an elastic solid, the Cauchy stress tensor $S$ is of the form

$$
S(X, t)=\hat{S}(F(X, t), X), \quad \forall X \in B
$$

where $\hat{S}: \operatorname{End}(V) \times B \rightarrow \operatorname{End}(V)$ is a function, called the stress response function, that depends on the body's constitution; and $t$ of course represents time.

Now we can already see why there is a physically important fourth-order tensor. Let us for simplicity consider a homogeneous elastic solid, for which $\hat{S}(F, X)$ is independent of its second argument $X$. So: $S(X, t)=\hat{S}(F(X, t))$. Then we expect to use the tensor calculus (essentially: multi-variable calculus) to analyse how $S$ depends on its $\operatorname{argument} F$. So we consider the derivatives of the components of $\hat{S}$ with respect to the components of $F$. This gives a four-index quantity: it is indeed a fourth-order tensor, the elasticity tensor, which I write as E:

$$
E_{i j k l}:=\frac{\partial \hat{S}_{i j}}{\partial F_{k l}}
$$

\section{Conclusion: yet more extrinsicality}

It is clear that as regards physics, the discussion in Sections 3 to 5 has barely scratched the surface: there is much more to be said about continuum mechanics. The same goes, I submit, for philosophy. In particular, as regards pointillisme: for brevity, I have restricted my critique. I have set aside various kinds of, or sources of, extrinsicalityboth physical and philosophical. I will not now lift these restrictions, nor even repeat the list of them from Section 1.1-why flog a dead horse?

But as a final remark, I will mention that yet "more" extrinsicality ensues when contingent claims, in particular the laws of a theory, are allowed as implicit premises 
in the implications that make a property extrinsic. In other words: many properties that are intrinsic in philosophers' (kind of) sense are nomically very extrinsic. That is: their ascriptions, when taken together with laws, will have many implications for states of affairs beyond the property's instance. Let me mention two very striking examples, each with a reference.

(1): The position, and even the existence, of a classical point-particle is nomically extrinsic, when we consider how classical particles are constituted, by decoherence, from the quantum state; (Butterfield (2006a, Section 5)).

(2): The second example is purely classical, and concerns point-particles in a Newtonian world, interacting by instantaneous forces such as gravity; (the forces need not have an inverse-square dependence on distance). It is due to Schmidt $(1997,1998)$. He proves that a particle's trajectory in spacetime, over a finite time-interval (no matter how short!), completely determines its trajectory throughout all time - regardless of how the other particles are moving. Agreed, the theorem needs some assumptions; but they are entirely reasonable. ${ }^{6}$ Thus the innovation here, in comparison with the usual idea of determinism for Newtonian gravity, is as follows. Usually one specifies the positions and momenta of all the point-particles across all of space at an instant. But here, one uses the idea that in a finite time-interval (no matter how short!), the trajectory of the particle one is considering encodes information about all the other particles' influence on it: and then the theorem is that it in fact encodes enough information to determine the entire future and past trajectory.

Let us transcribe this result into our jargon of nomic extrinsicality. That is, let us spell out its consequences for how much is implied about the rest of the universe by a statement of the history of the particle(s) in a spacetime region - when the implication is allowed to use the laws of motion and the reasonable assumptions as implicit premises, together with the particular history. The result is very striking. Namely: the history of a particle in a spacetime region of finite but arbitrarily small extent is nomically as extrinsic as one could imagine it to be. For when taken together with the laws of motion and reasonable assumptions, this small patch of history implies the particle's entire history!

Acknowledgements:- I am very grateful to audiences in Cambridge, Melbourne, and at the ESF conference in Zeist, and to A. Caulton, W. Myrvold, M. Wilson and the editor, for helpful conversations and comments. I thank O. Gonzalo, A. Stuart and Cambridge University Press, for permission to reproduce Figures 1 to 3 from $A$ First Course in Continuum Mechanics, copyright 2008.

Trinity College

Cambridge CB2 1TQ

\footnotetext{
${ }^{6}$ Namely: (i) the number of particles is finite; (ii) there is a distance less than which no pair of particles ever approach each other; (iii) there is a speed that no particle ever exceeds; (iv) like (iii): there is an acceleration (and so on for higher derivatives) that no particle ever exceeds. Cf. Schmidt (1997, p 446), (1998, pp. 83-84).
} 


\section{$7 \quad$ References}

Belot, G. [1998]: 'Understanding Electromagnetism', British Journal for the Philosophy of Science, 49, pp. 531-555.

Butterfield, J. [2004]: 'On the Persistence of Homogeneous Matter', available at: physics/0406021: and at http://philsci-archive.pitt.edu/archive/00002381/

Butterfield, J. [2004a]: 'Between Laws and Models: some philosophical morals of Lagrangian mechanics'; available at: physics/0409030; philsci-archive.pitt.edu/archive/00001937/

Butterfield, J. [2004b]: 'Aspects of modality in analytical mechanics', in P. Weingartner and M. Stoeltzner (eds), Formale Teleologie und Kausalitat in der Physik, Mentis; pp. 160-198; available at: physics/0210081; philsci-archive.pitt.edu/archive/00001192 Butterfield, J. [2005]: 'On the Persistence of Particles', in Foundations of Physics, 35, pp. 233-269, available at: physics/0401112; philsci-archive.pitt.edu/archive/00001586/. Butterfield, J. [2006]: 'Against Pointillisme about Geometry', in Time and History: Proceedings of the 28th Ludwig Wittgenstein Symposium, 2006, ed. F. Stadler and M. Stöltzner, Ontos Verlag, pp. 181-222; available at:

http://philsci-archive.pitt.edu/archive/00002552/ or arxiv.org/abs/physics/0512063.

Butterfield, J. [2006a]: 'The Rotating Discs Argument Defeated', in British Journal for the Philosophy of Science 57, pp. 1-45; available at:

http://philsci-archive.pitt.edu/archive/00002382/

Butterfield, J. [2006b]: 'Against Pointillisme about Mechanics', in British Journal for the Philosophy of Science 57, pp. 709-753; available at:

http://philsci-archive.pitt.edu/archive/00002553/ or http://arxiv.org/abs/physics/0512064.

Casey, J. [1992]: 'The Principle of Rigidification', Archive for the History of the Exact Sciences 49, pp. 329-383.

Crowe, M. [1967]: A History of Vector Analysis, Notre Dame University Press; reprinted by Dover.

Esfeld, M. [2001]: Holism in Philosophy of Mind and Philosophy of Physics, Kluwer Academic.

Gonzalo, O. and Stuart, A. [2008]: A First Course in Continuum Mechanics, Cambridge University Press.

Gurtin, M. [1981]: An Introduction to Continuum Mechanics, Academic Press.

Healey, R. [1991]: 'Holism and Nonseparability', Journal of Philosophy 88, pp. 393421.

Holland, P. [1993]: The Quantum Theory of Motion: an account of the de BroglieBohm causal interpretation of quantum mechanics, Cambridge University Press.

Kellogg, O. [1954]: Foundations of Potential Theory, Dover reprint of a 1929 original published by J. Springer.

Lehmkuhl, D. [2010]: 'Mass-energy-momentum: only there because of spacetime?', forthcoming in British Journal for the Philosophy of Science; available at: http://philsciarchive.pitt.edu/archive/00005137/

Lewis, D. [1983]: 'Extrinsic Properties', Philosophical Studies, 44, pp. 197-200; reprinted in Lewis [1999]; page references to reprint. 
Lewis, D. [1986]: Philosophical Papers, volume II, New York NY: Oxford University Press.

Lewis, D. [1994]: 'Humean Supervenience Debugged', Mind, 103, p 473-490; reprinted in Lewis [1999], pp. 224-247.

Lewis, D. [1999]: Papers in Metaphysics and Epistemology, Cambridge University Press.

Marsden, J. and Hughes, T. (1983): Mathematical Foundations of Continuum Mechanics, Prentice-Hall; reprinted 1994 by Dover.

Myrvold, W. [2002]: 'On Peaceful Coexistence: Is the Collapse Postulate Incompatible with Relativity?', Studies in History and Philosophy of Modern Physics 33, pp. 435466.

Myrvold, W. [2003]: 'Relativistic quantum becoming', British Journal for the Philosophy of Science 53, pp. 475-500 ; available at: http://philsci-archive.pitt.edu/archive/00000569/ Myrvold, W. [2009]: 'Chasing chimeras', in British Journal for the Philosophy of Science 60, pp. 635-646; available at: http://philsci-archive.pitt.edu/archive/00004529/ Myrvold, W. [2010]: 'Non-Separability, classical and quantum', forthcoming in British Journal for the Philosophy of Science; available at:

http://philsci-archive.pitt.edu/archive/00004859/

Schmidt, J. [1997], 'Classical Universes are perfectly predictable', Studies in the History and Philosophy of Modern Physics 28B, pp. 433-460.

Schmidt, J. [1998], 'Predicting the motion of particles in Newtonian mechanics and special relativity', Studies in the History and Philosophy of Modern Physics 29B, pp. 81-122.

Smith, S. [2007]: 'Continuous bodies, impenetrability and contact interactions: the view from the applied mathematics of continuum mechanics', British Journal for the Philosophy of Science $\mathbf{5 8}$, pp. 503-538.

Teller, P. [1986], 'Relational holism and quantum mechanics', British Journal for the Philosophy of Science $\mathbf{3 7}$, pp. 71-81.

Truesdell, C. [1991]: A First Course in Rational Continuum Mechanics, volume 1, second edition, Academic.

Wilson, M [1998]: 'Classical Mechanics'; Entry in The Routledge Encyclopedia of Philosophy, ed. E. Craig, Routledge; pp. 251-259.

Wilson, M [2006]: Wandering Significance: an essay on conceptual behaviour, Oxford University Press. 